\title{
Time to Understand More about Spontaneous Regression of Cancer
}

\author{
Tamar Tadmor ${ }^{a, b}$ \\ ${ }^{a}$ Hematology Unit, Bnai Zion Medical Center, Haifa, Israel; ${ }^{b}$ The Ruth and Bruce Rappaport Faculty of \\ Medicine, Technion, Haifa, Israel
}

In the first issue of Acta Haematologica in 2019, Pasvolsky et al. [1] describe a 32-year-old woman diagnosed in June 2014 as having classical Hodgkin lymphoma (cHL) nodular sclerosis type stage IIA. The diagnosis was based on the presence of an enlarged left cervical lymph node. Chemotherapy with the ABVD regimen was planned but the patient refused treatment. She has been followed over the past 4 years and her current PET-CT scan shows no uptake at any of the lymph node sites originally described at diagnosis, suggesting a spontaneous remission of her disease.

The first question to be asked in this case would probably relate to "best prognosis is the wrong diagnosis" and whether the patient truly had cHL? In this regard, the authors themselves confirmed that the biopsy was indeed examined by another, independent expert hematopathologist, who agreed with the diagnosis of cHL. It therefore does not appear to have been a "misdiagnosis."

Cases of spontaneous regression of neoplastic disease are rare, but we should be aware of this phenomenon. A PubMed search using the terms "spontaneous regression and cancer" yields hundreds of publications. Furthermore, many practicing hematologists and oncologists have probably encountered, at some point, individual patients who refused treatment and "survived" despite the fact that they did not receive a specific therapy, and they are still doing well. It is also of interest that some of these

\section{KARGER}

() 2019 S. Karger AG, Basel

E-Mail karger@karger.com

www.karger.com/aha patients have experienced recurrent cycles of spontaneous remission and relapse [2], showing that further spontaneous remissions of untreated cancer might occur.

The spontaneous healing of cancer is a phenomenon that has been observed and reported for several years by oncologists, hematologists, pathologists, and researchers [3]. One of the pioneer researchers in the field, Dr. Everson [4], clearly defined this phenomenon in one of his publications in 1968 "as the partial or complete disappearance of a malignant tumor, in the absence of all treatment or in the presence of therapy which is considered inadequate to exert a significant influence on neoplastic disease." He also emphasized the fact that these cases of cancer were all diagnosed by histopathological examination of a tissue biopsy [4].

Several hypotheses to explain this phenomenon have been published [3-6]. Most of them propose theories relating to a trigger mechanism which stimulates the host innate immune system to generate an "autoimmune type immunotherapy" against tumor cells. However, the main challenge would still be to identify the exact nature of this trigger.

Editorial comment on "Spontaneous regression of Hodgkin lymphoma: case report and review of the literature" by Pasvolsky et al. [Acta Haematol 2019;141:14-18, DOI: 10.1159/000494422]. 
A possible role for immune stimulation has been attributed to the process of active infection, which induces tumor regression [5, 6]. In 1891, William Coley, a surgeon from New York's Memorial Hospital, described the clinical course of one of his patients who had a progressive sarcoma that regressed totally during treatment for erysipelas caused by Streptococcus pyogenes. Coley suspected that the infection was somehow responsible for the "miraculous cure." He later realized that the patient's activated immunity in response to the acute infection was the key factor in the regression of the cancer $[5,6]$. After this observation, Dr. Coley started to study the relationship between infection and tumor regression, aiming to develop an antibacterial vaccine [4]. The vaccine used contained 2 inactivated bacteria, S. pyogenes and Serratia marcescens, and was reported on in the early 19th century as "Coley's toxins."

This link between tumor regression and infection has also been associated more recently with "oncolytic" viruses $[4,5]$. These viruses have the capacity to promote tumor cell death by inducing the lysis of infected cells, thereby exposing tumor-associated antigens, neoantigens, and other signals that may subsequently trigger an antitumor immune response. Several classes of viruses are now under investigation, including a modified Herpes simplex virus 1, Coxsackievirus, Vaccinia virus, Adenovirus, Reovirus, the Newcastle disease virus, the measles virus, and others $[4,5]$.

However, not all reported cases of spontaneous regression of cancer describe a link with infection, as is the case in the report by Pasvolsky et al. [1]; the patient did not have a history of fever or any infectious complications. Another possible explanation could involve an inflammatory reaction unrelated to infection, which, theoretically, could also affect immune surveillance and activate a cascade of events involving cytotoxic $\mathrm{T}$ lymphocytes which can initiate an antitumor cell immune response [5]. Other stimuli reported include the use of several drugs such as antihypertensive calcium blockers, supplements, and dietary ingredients like green tea [6]. One could also speculate on the possible association of "body and mind" [7], a difficult topic to study or prove.

In conclusion, it is evident that the phenomenon of spontaneous regression of cancer in general (and cHL in particular) does exist and is worthy of more investigation in the future. Identifying the mechanism underlying this phenomenon is important and would help in the development of targeted immunotherapies that could serve as treatment options for patients with $\mathrm{cHL}$ and other cancers.

\section{Disclosure Statement}

The author has no conflicts of interest to declare.

\section{Funding Sources}

No funding was used.

\section{References}

1 Pasvolsky O, Berger T, Bernstine H, Hayman L, Raanani P, Vidal L. Spontaneous Regression of Hodgkin Lymphoma: Case Report and Review of the Literature. Acta Haematol. 2019;141(1):14-8.

2 Kaufmann Y, Many A, Rechavi G, Mor O, Biniaminov M, Rosenthal E, et al. Brief report: lymphoma with recurrent cycles of spontaneous remission and relapse-possible role of apoptosis. N Engl J Med. 1995 Feb;332(8): 507-10.
3 Jessy T. Immunity over inability: the spontaneous regression of cancer. J Nat Sci Biol Med. 2011 Jan;2(1):43-9.

4 Everson TC. Spontaneous regression of cancer. Ann N Y Acad Sci. 1964 Apr;114(2):721-35.

5 Grivennikov SI, Greten FR, Karin M. Immunity, inflammation, and cancer. Cell. 2010 Mar;140(6):883-99.
6 Herishanu Y, Solar I, Ben-Ezra J, Cipok M, Meirsdorf S, Amariglio N, et al. Complete spontaneous regression of chronic lymphocytic leukemia. J Clin Oncol. 2012 Sep; 30(26):e254-6.

7 Challis GB, Stam HJ. The spontaneous regression of cancer. A review of cases from 1900 to 1987. Acta Oncol. 1990;29(5):545-50. 